\title{
Does Self-rated Attractiveness Predict Women's Preferences for Facial Masculinity? Data From an Arab Sample
}

\author{
Sarah A. H. Alharbi $^{1}$ • Iris J. Holzleitner ${ }^{1}$ • S. Adil Saribay ${ }^{2}$. \\ Benedict C. Jones ${ }^{3}$ (D) Anthony J. Lee ${ }^{4}$
}

Received: 25 November 2020 / Revised: 12 February 2021 / Accepted: 15 February 2021/

Published online: 8 March 2021

(C) Crown 2021

\section{Abstract}

Objectives Because more attractive women may be better able to attract and/or retain masculine mates, many researchers have proposed that women who consider themselves to be more physically attractive will show stronger preferences for men displaying masculine facial characteristics. Empirical evidence for this putative association between women's self-rated attractiveness and preference for facial masculinity has come almost entirely from studies of Western women. Thus, we investigated whether this pattern of results also occurs in a sample of non-Western women.

Methods We investigated the relationship between self-rated attractiveness and facialmasculinity preferences in a sample of Arab women $(\mathrm{N}=281)$. Facial-masculinity preferences were assessed from attractiveness judgments of masculinized versus feminized versions of face images.

Results By contrast with previous findings for Western women's self-rated attractiveness, we observed no compelling evidence that Arab women who considered themselves to be more attractive showed stronger preferences for masculine men.

Conclusions Our results suggest that previously reported associations between selfrated attractiveness and masculinity preferences might be somewhat culture specific, potentially reflecting cultural differences in typical mating strategies.

Keywords facial attractiveness $\cdot$ mate preferences $\cdot$ cultural differences $\cdot$ individual differences $\cdot$ condition-dependent preferences $\cdot$ market value

Benedict C. Jones

benedict.jones@strath.ac.uk

Extended author information available on the last page of the article 


\section{Introduction}

Masculine characteristics in men's faces are commonly assumed to be positively correlated with indices of heritable aspects of male health and physical strength, but negatively correlated with indices of men's willingness to invest resources in mates and offspring (Little et al. 2001; Penton-Voak et al. 2003). Although evidence that facial masculinity is a valid cue of men's health is mixed (see Jones et al. 2019 for a detailed discussion of these issues), there is compelling evidence that masculine characteristics in men's faces are positively correlated with measures of their upper body strength and threat potential (Han et al. 2017; Holzleitner et al. 2014; Windhager et al. 2011). Furthermore, increasing masculine characteristics in images of men's faces decreases perceptions of their prosociality (Perrett et al. 1998). Consequently, many researchers have proposed that the extent to which women prefer masculine men over feminine men as potential mates depends, at least in part, on how they weight the benefits of choosing a healthy, physically strong partner versus the benefits of choosing of a prosocial partner (Little et al. 2001; Penton-Voak et al. 2003). How individual women resolve this trade-off is thought to be influenced by aspects of their personal circumstances, such as the harshness of the environment in which they live, their position in the menstrual cycle, and their perceptions of their own physical attractiveness (Holzleitner and Perrett 2017; Little et al. 2001; Penton-Voak et al. 2003).

Evidence for this trade-off account of systematic variation in women's masculinity preferences is mixed. For example, some studies have found that women in harsher environments prefer men with more masculine faces (DeBruine et al. 2010, 2011), other studies have found that women in harsher environments prefer men with less masculine faces (Marcinkowska et al. 2019; Scott et al. 2014), while other studies have found little evidence that environmental harshness influences women's preferences for masculine men (Dixson et al. 2017). Similarly, while some studies have found that women show stronger preferences for men with masculine faces during the most fertile phase of their menstrual cycle (e.g., Little and Jones 2012; Penton-Voak et al. 1999), other studies observed no compelling evidence that women's preferences for men with masculine faces tracked changes in fertility during the menstrual cycle (e.g., Jones et al. 2018; Marcinkowska et al. 2018).

By contrast with the mixed findings for the effects of environmental harshness and fertility described above, findings from studies of the relationship between women's perceptions of their own physical attractiveness (i.e., their self-rated attractiveness) and preferences for men with masculine facial characteristics appear to be much more reliable. Many studies of systematic variation in women's mate preferences have now reported that women who rated themselves as more physically attractive show stronger preferences for men with masculine facial characteristics (Batres et al. 2020; Docherty et al. 2020; Kandrik and DeBruine 2012; Little et al. 2001; but see also Clarkson et al. 2020; Ekrami et al. 2020; Penton-Voak et al. 2003; Zietsch et al. 2015). This association between self-rated attractiveness and masculinity preferences is thought to occur because women who consider themselves to be more attractive believe they will be able to attract, retain, and/or replace masculine romantic partners (i.e., believe that the potential costs of choosing of a masculine mate are less pronounced for more attractive women, Batres et al. 2020; Docherty et al. 2020; Kandrik and DeBruine 2012; Little et al. 2001). Indeed, research in which women's perceptions of their own attractiveness 
were experimentally manipulated found that increasing women's perceptions of their own attractiveness increases their preferences for men displaying masculine facial characteristics (Little and Mannion 2006).

Previous studies reporting a positive relationship between women's self-rated attractiveness and their preferences for masculine characteristics in men's faces have investigated this issue in samples of women living in Western cultures (Batres et al. 2020; Docherty et al. 2020; Kandrik and DeBruine 2012; Little et al. 2001). This is potentially problematic, since the evolutionary explanation posited for this effect implies that self-rated attractiveness will influence women's masculinity preferences across diverse cultures. Since only Western samples have been tested, we are unable to tell whether this effect would generalize to non-Western samples or is unique to Western cultures. Thus, we investigated the relationship between self-rated attractiveness and preferences for masculinized versus feminized versions of men's faces in a sample of Arab women in order to test the generalizability of the effect. Because some studies have reported that Western women who consider themselves to be more attractive show stronger preferences for femininity in women's faces (Docherty et al. 2020; Kandrik and DeBruine 2012), we also assessed Arab women's preferences for masculinized versus feminized versions of women's faces.

\section{Methods}

\section{Participants}

Two hundred and eighty-one Arab women (mean age $=24.96$ years, $\mathrm{SD}=6.62$ years) participated in this online study (faceresearch.org, DeBruine 2019). Participants were recruited using links to an online study of face perceptions on Saudi Arabian social media and were from Saudi Arabia, Algeria, Libya, Jordan, Oman, Egypt, and Kuwait. All participants provided informed consent and the study was approved by the Psychology Ethics Committee, University of Glasgow.

\section{Stimuli}

Stimuli were manufactured using methods used in previous studies on this topic (e.g., DeBruine et al. 2010; Jones et al. 2018; Perrett et al. 1998), from an open access set of Turkish face images (Saribay et al. 2018), and using standard computer graphic methods (DeBruine 2018; Tiddeman et al. 2001). Eleven images of people wearing glasses or headscarves that obscured the face were removed from the image set.

First, we manufactured a female prototype by averaging the shape, color, and texture information from 142 female images. Next, we manufactured a male prototype by averaging the shape, color, and texture information from 111 male images. Finally, we created masculinized and feminized versions of 60 of the individual face images (30 male, 30 female) by adding or subtracting $50 \%$ of the differences in 2D shape between the male and female prototypes to each of 60 individual faces randomly selected from the full image set. Manipulated prototypes are shown in Fig. 1.

This process created 30 male and 30 female pairs, with each pair consisting of a masculinized and feminized version of a given face. These stimuli have been used in two 
other recent studies of Arab women's face preferences (Alharbi et al. 2020a, b). None of the women tested in the current study had participated in these previous studies.

\section{Procedure}

The 60 pairs of faces were shown in a fully randomized order, with the side of the screen on which the masculinized and feminized versions were presented also fully randomized. Participants were instructed (in Arabic) to click on the face in each pair that looked more attractive. Each woman also rated their own physical attractiveness using a 1 (very unattractive) to 7 (very attractive) scale $(\mathrm{M}=5.11, \mathrm{SD}=1.21)$.

Forced-choice paradigms can produce qualitatively different patterns of results to other methods for assessing perceptions of faces (Jones and Jaeger 2019). We used the forced choice method in the current study to allow our results to be directly compared with previous research investigating putative links between self-rated attractiveness and face preferences.
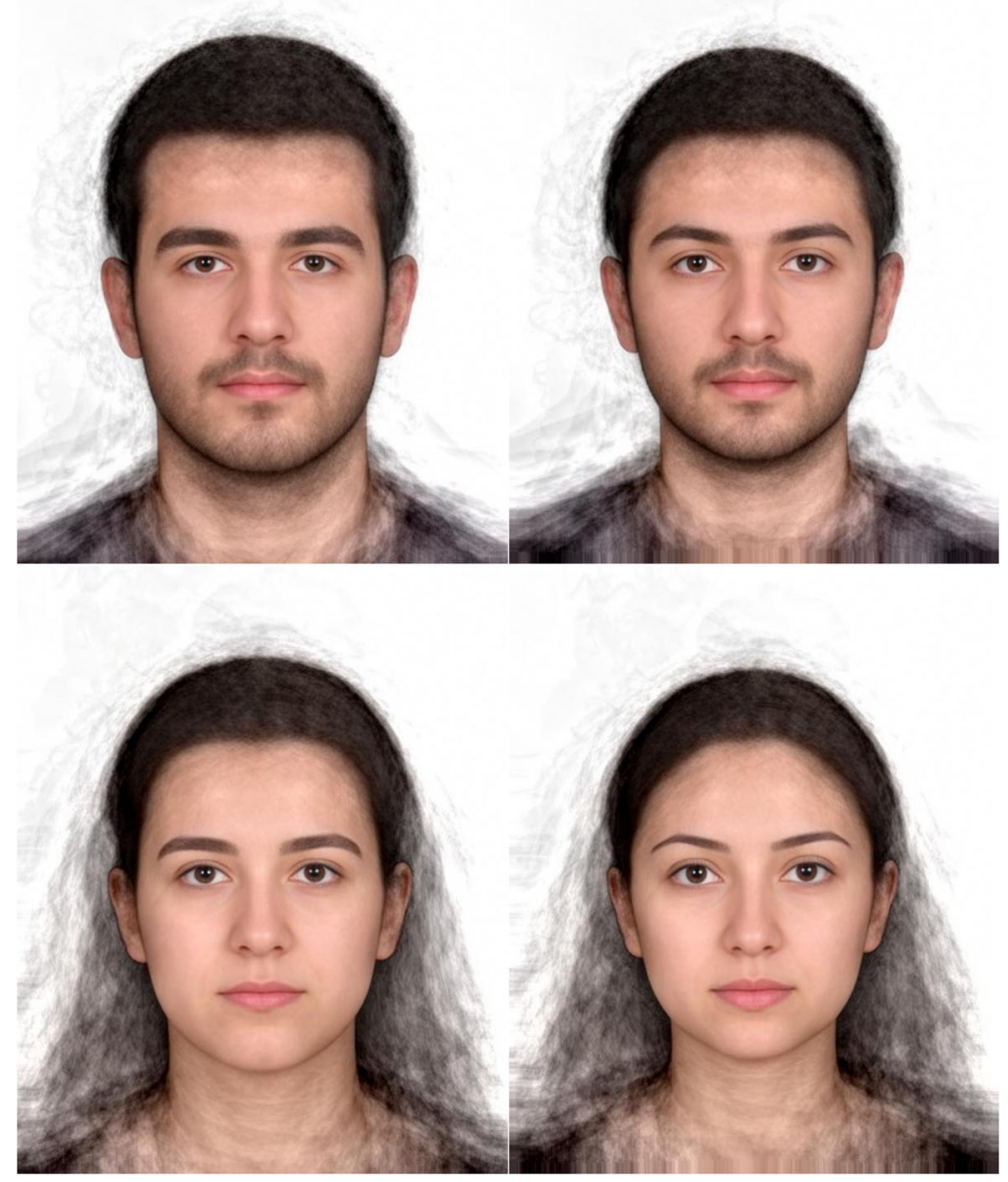

Fig. 1 Examples of the sexual dimorphism transform applied to male (top row) and female (bottom row) face prototypes. Masculinized versions are shown in the left column and feminized versions in the right column. 


\section{Results}

Data, output, and analysis code are available at https://osf.io/gp8bk/. Responses were analyzed with binomial mixed effects models using the lme4 (Bates et al. 2015) and lmerTest (Kuznetsova et al. 2015) package in the R software (R Core Team 2013). Participant's choice was the dependent variable (chose masculinized $=0$, chose feminized $=1$ ). Participant age and self-rated attractiveness (both z-standardized at the participant level) and sex of face (effect coded: male $=-0.5$, female $=0.5$ ) were predictors. Random slopes were specified maximally (Barr et al. 2013) with participant ID and stimulus ID as the grouping variables. Results are summarized in Fig. 2.

In this initial analysis, the intercept was significant and positive (estimate $=0.69$, $\mathrm{SE}=0.11, \mathrm{z}=6.02, \mathrm{p}<.001)$. Converting this estimate to proportions, this equates to women choosing, on average, the feminized versions as the more attractive face on $66 \%$ of trials. Neither the main effects of participant age (estimate $=0.09, \mathrm{SE}=0.06$, $\mathrm{z}=1.60, \mathrm{p}=.10$ ) or self-rated attractiveness (estimate $=0.01, \mathrm{SE}=0.06, \mathrm{z}=0.20$, $\mathrm{p}=.84)$ were significant. However, the main effect of sex of face was significant (estimate $=0.73, \mathrm{SE}=0.20, \mathrm{z}=3.59, \mathrm{p}<.001$ ).

When male and female faces were analyzed separately, the intercept was significant for female faces (estimate $=1.05, \mathrm{SE}=0.16, \mathrm{z}=6.60, \mathrm{p}<.001$ ) and male faces (estimate $=0.32, \mathrm{SE}=0.15, \mathrm{z}=2.18, \mathrm{p}=.029$ ). This equates to, on average, women choosing the feminized version as the more attractive face on $74 \%$ of female-face trials and $58 \%$ of male-face trials. The effect of participant age was not significant for female faces (estimate $=0.01, \mathrm{SE}=0.06, \mathrm{z}=0.04, \mathrm{p}=.96$ ), but was significant for male faces (estimate $=0.18, \mathrm{SE}=0.06, \mathrm{z}=2.65, \mathrm{p}=.008$ ). The effect of self-rated attractiveness was not significant for female faces (estimate $=0.03, \mathrm{SE}=0.07, \mathrm{z}=0.44, \mathrm{p}=.65$ ) or male faces (estimate $=-0.01, \mathrm{SE}=0.07, \mathrm{z}=-0.09, \mathrm{p}=.92$ ).

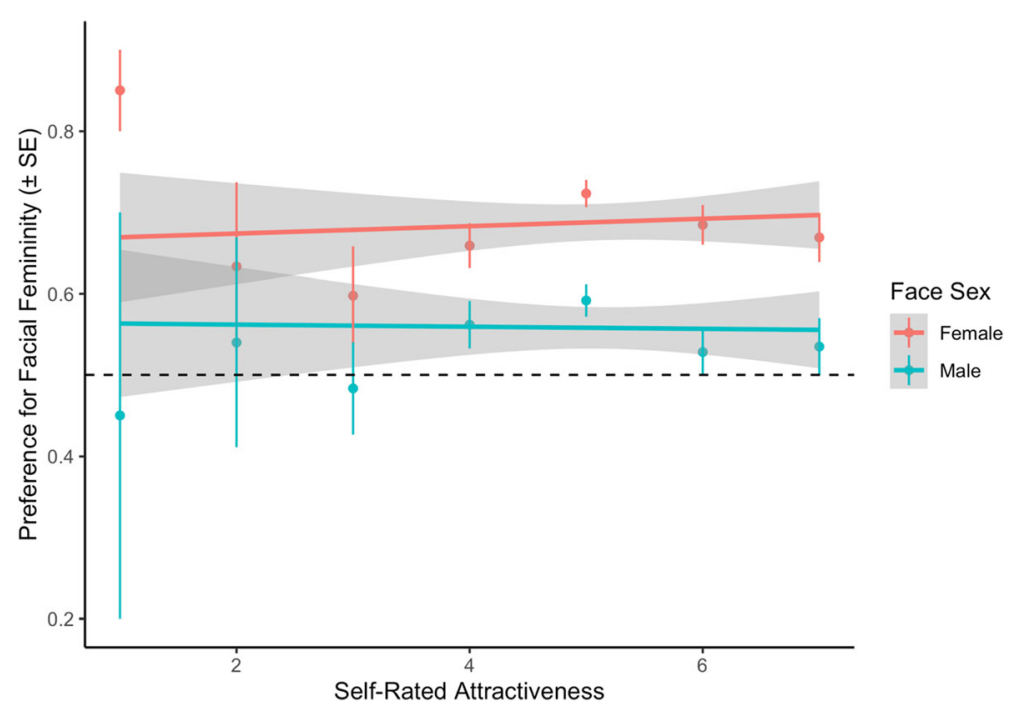

Fig. 2 The non-significant relationships between self-rated attractiveness and femininity preferences. Points and error bars show mean and SE respectively. The grey band shows the $95 \%$ CI for the regression lines. 


\section{Discussion}

We found that, on average, Arab women considered feminized versions of both male and female faces to be more attractive than masculinized versions. This pattern of results replicates preferences for femininity when Arab women judged the attractiveness of masculinized versus feminized versions of Arab men's and women's faces in previous research (Alharbi et al. 2020a). However, we observed no evidence that Arab women who rated themselves as more attractive showed stronger preferences for masculine men or feminine women. Thus, our results for self-rated attractiveness do not replicate those previously reported for Western women in which women who rated themselves as more attractive showed stronger preferences for masculine men (Batres et al. 2020; Docherty et al. 2020; Kandrik and DeBruine 2012; Little et al. 2001) and feminine women (Docherty et al. 2020; Kandrik and DeBruine 2012).

Given that self-rated attractiveness appears to reliably predict women's preferences for men displaying masculine facial characteristics in studies of Western women's face preferences (Batres et al. 2020; Docherty et al. 2020; Kandrik and DeBruine 2012; Little et al. 2001), it is useful for us to discuss some of the possible explanations of why this effect of self-rated attractiveness on face preferences does not appear to generalize to Arab women's face preferences.

First, might the different patterns of results reported for self-rated attractiveness and face preferences in samples of Western and Arab women reflect methodological differences among studies? We suggest that this explanation is unlikely because the methods we used to manipulate masculinity in face images, the paradigm we used to assess masculinity preferences, and the methods we used to assess self-rated attractiveness are identical to those used in studies of Western women's face preferences.

Second, might Arab women ascribe qualitatively different traits to masculine men than Western women do? Previous research on Arab women's perceptions of men's age and dominance have reported results that are strikingly similar to those reported in research on Western women's perceptions of men's age and dominance (i.e., both Western and Arab women judge masculinized versions of men's faces to be older and more dominant looking than feminized versions, Boothroyd et al. 2007; Alharbi et al. $2020 \mathrm{a}, \mathrm{b})$. Thus, it is unlikely that we did not observe a significant correlation between self-rated attractiveness and masculinity preferences in the current study because masculinity has different effects on Arab and Western women's perceptions of men's age and dominance. However, while it is well established that Western women perceive masculinized versions of men's faces to look less trustworthy than feminized versions (e.g., Perrett et al. 1998), this does not appear to be the case for Arab women (Alharbi et al. 2020a). If perceptions of trustworthiness play an important role in the link between self-rated attractiveness and masculinity preferences, cultural differences in the extent to which women perceive masculine men to be untrustworthy might explain why self-rated attractiveness predicts masculinity preferences in samples of Western, but not Arab, women.

A third possibility is that self-rated attractiveness predicts masculinity preferences in samples of Western, but not Arab, women, because of differences in the mating strategies typical for such women. Indeed, previous research has shown that Arab women are, on average, less open to uncommitted sexual relationships than Western women (Marcinkowska et al. 2019). Some studies have also reported that Western 
women tend to show stronger preferences for masculinity when assessing men's attractiveness for short-term relationships (Jones et al. 2018; Little et al. 2001; but see also Dixson et al. 2018 and Stower et al. 2020) and that Western women who score higher on measures of openness to uncommitted sexual relationships tend to be more physically attractive (Fisher et al. 2016; Perilloux et al. 2013) and show stronger preferences for masculine men (Stower et al. 2020; Zietsch et al. 2015). Although these effects are typically quite small, such results potentially implicate individual differences in sociosexuality in the association between women's self-rated attractiveness and masculinity preferences.

In conclusion, we observed no evidence that Arab women who rate their own physical attractiveness to be particularly high show stronger preferences for masculine characteristics in men's faces. This contrasts markedly with findings from previous studies of Western women's face preferences, in which self-rated attractiveness tended to reliably predict women's masculinity preferences. Thus, our null result for Arab women's self-rated attractiveness suggests the effect of self-rated attractiveness on masculinity preferences may be somewhat culture specific. Further work investigating the association between self-rated attractiveness and masculinity preferences in more culturally diverse samples would be needed to clarify the cultural and ecological factors that determine the extent to which self-rated attractiveness predicts masculinity preferences among cultures.

Funding S Adil Saribay was supported by the Czech Science Foundation project regno 18-10298S

Declarations The study was performed in accordance with UK ethical standards and all participants provided informed consent.

Conflict of Interest The authors declare that they have no conflict of interest.

Open Access This article is licensed under a Creative Commons Attribution 4.0 International License, which permits use, sharing, adaptation, distribution and reproduction in any medium or format, as long as you give appropriate credit to the original author(s) and the source, provide a link to the Creative Commons licence, and indicate if changes were made. The images or other third party material in this article are included in the article's Creative Commons licence, unless indicated otherwise in a credit line to the material. If material is not included in the article's Creative Commons licence and your intended use is not permitted by statutory regulation or exceeds the permitted use, you will need to obtain permission directly from the copyright holder. To view a copy of this licence, visit http://creativecommons.org/licenses/by/4.0/.

\section{References}

Alharbi, S. A., Holzleitner, I. J., Lee, A. J., Saribay, S. A., \& Jones, B. C. (2020a). Women's preferences for sexual dimorphism in faces: Data from a sample of Arab women. Evolutionary Psychological Science, 6, 328-334.

Alharbi, S. A., Holzleitner, I. J., Lee, A. J., Saribay, S. A., \& Jones, B. C. (2020b). Facial masculinity increases perceptions of men's age, but not perceptions of their health: Data from an Arab sample. Evolutionary Psychological Science, online.

Barr, D. J., Levy, R., Scheepers, C., \& Tily, H. J. (2013). Random effects structure for confirmatory hypothesis testing: Keep it maximal. Journal of Memory and Language, 68(3), 255-278. 
Bates, D., Mächler, M., Bolker, B. M., \& Walker, S. C. (2015). Fitting linear mixed-effects models using lme4. Journal of Statistical Software, 67(1), 1-48.

Batres, C., Jones, B. C., \& Perrett, D. I. (2020). Attraction to men and women predicts sexual dimorphism preferences. International Journal of Sexual Health.

Boothroyd, L. G., Jones, B. C., Burt, D. M., \& Perrett, D. I. (2007). Partner characteristics associated with masculinity, health and maturity in male faces. Personality and Individual Differences, 43, 1161-1173.

Clarkson, T. R., Sidari, M. J., Sains, R., Alexander, M., Harrison, M., Mefodeva, V., ... Dixson, B. J. (2020). A multivariate analysis of women's mating strategies and sexual selection on men's facial morphology. Royal Society Open Science, 7(1), 191209.

DeBruine, L. M. (2018). debruine/webmorph: Beta release 2 (Version v0.0.0.9001). Zenodo. https://doi.org/ 10.5281/zenodo.1162670.

DeBruine, L. M. (2019). debruine/experimentum: Beta release 1 (Version v.0.1). Zenodo. https://doi.org/10. 5281/zenodo.2634356.

DeBruine, L. M., Jones, B. C., Crawford, J. R., Welling, L. L., \& Little, A. C. (2010). The health of a nation predicts their mate preferences: Cross-cultural variation in women's preferences for masculinized male faces. Proceedings of the Royal Society B: Biological Sciences, 277, 2405-2410.

DeBruine, L. M., Jones, B. C., Little, A. C., Crawford, J. R., \& Welling, L. L. (2011). Further evidence for regional variation in women's masculinity preferences. Proceedings of the Royal Society B: Biological Sciences, 278(1707), 813-814.

Dixson, B. J., Blake, K. R., Denson, T. F., Gooda-Vossos, A., O'Dean, S. M., Sulikowski, D., ... Brooks, R. C. (2018). The role of mating context and fecundability in women's preferences for men's facial masculinity and beardedness. Psychoneuroendocrinology, 93, 90-102.

Dixson, B. J., Little, A. C., Dixson, H. G., \& Brooks, R. C. (2017). Do prevailing environmental factors influence human preferences for facial morphology? Behavioral Ecology, 28, 1217-1227.

Docherty, C., Lee, A. J., Hahn, A. C., DeBruine, L. M., \& Jones, B. C. (2020). Do more attractive women show stronger preferences for male facial masculinity? Evolution and Human Behavior. online.

Ekrami, O., Claes, P., Shriver, M. D., Weinberg, S. M., Marazita, M. L., Walsh, S., \& Van Dongen, S. (2020). Effects of male facial masculinity on perceived attractiveness. Adaptive Human Behavior and Physiology, online.

Fisher, C. I., Hahn, A. C., DeBruine, L. M., \& Jones, B. C. (2016). Is women's sociosexual orientation related to their physical attractiveness? Personality and Individual Differences, 101, 396-399.

Han, C., Kandrik, M., Hahn, A. C., Fisher, C. I., Feinberg, D. R., Holzleitner, I. J., ... Jones, B. C. (2017). Interrelationships among men's threat potential, facial dominance, and vocal dominance. Evolutionary Psychology, 15(1), 1474704917697332.

Holzleitner, I. J., Hunter, D. W., Tiddeman, B. P., Seck, A., Re, D. E., \& Perrett, D. I. (2014). Men's facial masculinity: When (body) size matters. Perception, 43(11), 1191-1202.

Holzleitner, I. J., \& Perrett, D. I. (2017). Women's preferences for men's facial masculinity: trade-off accounts revisited. Adaptive Human Behavior and Physiology, 3(4), 304-320.

Jones, A. L., \& Jaeger, B. (2019). Biological bases of beauty revisited: The effect of symmetry, averageness, and sexual dimorphism on female facial attractiveness. Symmetry, 11(2), 279.

Jones, B. C., Hahn, A. C., \& DeBruine, L. M. (2019). Ovulation, sex hormones, and women's mating psychology. Trends in Cognitive Sciences, 23(1), 51-62.

Jones, B. C., Hahn, A. C., Fisher, C. I., Wang, H., Kandrik, M., Han, C., \& O’Shea, K. J. (2018). No compelling evidence that preferences for facial masculinity track changes in women's hormonal status. Psychological Science, 29, 996-1005.

Kandrik, M., \& DeBruine, L. M. (2012). Self-rated attractiveness predicts preferences for opposite-sex faces, while self-rated sex-typicality predicts preferences for same-sex faces. Journal of Evolutionary Psychology, 10, 177-186.

Kuznetsova, A., Brockhoff, P. B., \& Christensen, R. H. B. (2015). lmerTest: Tests for random and fixed effects for linear mexed effect models. Retrieved from https:/CRAN.R-project.org/package=lmerTest.

Little, A. C., Burt, D. M., Penton-Voak, I. S., \& Perrett, D. I. (2001). Self-perceived attractiveness influences human female preferences for sexual dimorphism and symmetry in male faces. Proceedings of the Royal Society B: Biological Sciences, 268, 39-44.

Little, A. C., \& Jones, B. C. (2012). Variation in facial masculinity and symmetry preferences across the menstrual cycle is moderated by relationship context. Psychoneuroendocrinology, 37(7), 999-1008.

Little, A. C., \& Mannion, H. (2006). Viewing attractive or unattractive same-sex individuals changes self-rated attractiveness and face preferences in women. Animal Behaviour, 72, 981-987. 
Marcinkowska, U. M., Galbarczyk, A., \& Jasienska, G. (2018). La donna è mobile? Lack of cyclical shifts in facial symmetry, and facial and body masculinity preferences-A hormone based study. Psychoneuroendocrinology, 88, 47-53.

Marcinkowska, U. M., Rantala, M. J., Lee, A. J., Kozlov, M. V., Aavik, T., Cai, H., \& Onyishi, I. E. (2019). Women's preferences for men's facial masculinity are strongest under favorable ecological conditions. Scientific Reports, 9(1), 1-10.

Penton-Voak, I. S., Little, A. C., Jones, B. C., Burt, D. M. Tiddeman, B. P., \& Perrett, D. I. (2003). Female condition influences preferences for sexual. dimorphism in faces of male humans (Homo sapiens). Journal of Comparative Psychology, 117, 264-271.

Penton-Voak, I. S., Perrett, D. I., Castles, D. L., Kobayashi, T., Burt, D. M., Murray, L. K., \& Minamisawa, R. (1999). Menstrual cycle alters face preference. Nature, 399(6738), 741-742.

Perilloux, C., Cloud, J. M., \& Buss, D. M. (2013). Women's physical attractiveness and short-term mating strategies. Personality and Individual Differences, 54(4), 490-495.

Perrett, D. I., Lee, K. J., Penton-Voak, I., Rowland, D., Yoshikawa, S., Burt, D. M., \& Akamatsu, S. (1998). Effects of sexual dimorphism on facial attractiveness. Nature, 394(6696), 884-887.

R Core Team. (2013). A language and environmental for statistical computing. Vienna: R Foundation for Statistical Computing.

Saribay, S. A., Biten, A. F., Meral, E. O., Aldan, P., Třebický, V., \& Kleisner, K. (2018). The Bogazici face database: Standardized photographs of Turkish faces with supporting materials. PLoS One,13(2), e0192018.

Scott, I. M., Clark, A. P., Josephson, S. C., Boyette, A. H., Cuthill, I. C., Fried, R. L., \& Honey, P. L. (2014). Human preferences for sexually dimorphic faces may be evolutionarily novel. Proceedings of the National Academy of Sciences, 111(40), 14388-14393.

Stower, R. E., Lee, A. J., McIntosh, T. L., Sidari, M. J., Sherlock, J. M., \& Dixson, B. J. (2020). Mating strategies and the masculinity paradox: how relationship context, relationship status, and sociosexuality shape women's preferences for facial masculinity and beardedness. Archives of Sexual Behavior, 49, 809820 .

Tiddeman, B., Burt, M., \& Perrett, D. (2001). Prototyping and transforming facial textures for perception research. IEEE Computer Graphics and Applications, 21, 42-50.

Windhager, S., Schaefer, K., \& Fink, B. (2011). Geometric morphometrics of male facial shape in relation to physical strength and perceived attractiveness, dominance, and masculinity. American Journal of Human Biology, 23(6), 805-814.

Zietsch, B. P., Lee, A. J., Sherlock, J. M., \& Jern, P. (2015). Variation in women's preferences regarding male facial masculinity is better explained by genetic differences than by previously identified contextdependent effects. Psychological Science, 1-9.

Publisher's Note Springer Nature remains neutral with regard to jurisdictional claims in published maps and institutional affiliations.

\section{Affiliations}

\section{Sarah A. H. Alharbi ${ }^{1}$ • Iris J. Holzleitner ${ }^{1}$ • S. Adil Saribay ${ }^{2} \cdot$ Benedict C. Jones $^{3}$. Anthony J. Lee ${ }^{4}$}

1 Institute of Neuroscience \& Psychology, University of Glasgow, Glasgow, UK

2 Department of Psychology, Kadir Has Üniversitesi, Istanbul, Turkey

3 School of Psychological Sciences and Health, University of Strathclyde, Glasgow, UK

4 Division of Psychology, University of Stirling, Stirling, UK 